\title{
High-energy isolated attosecond pulses generated by above-saturation few-cycle fields
}

\author{
F. Ferrarił, F. Calegarił, M. Lucchini", C. Vozzi, S. Stagira, G. Sansone and M. Nisoli^
}

\begin{abstract}
The applications of isolated attosecond pulses reported to date, which have demonstrated the great potential of attosecond technology in the investigation of ultrafast electronic processes, have been limited by the low photon flux of the available attosecond sources. We report on the generation of isolated sub-160-as pulses (at a photon energy of $\sim 30 \mathrm{eV}$ ) with a pulse energy, on target, of a few nanojoules. The efficient generation of isolated attosecond pulses in noble gases is produced by $\mathbf{5 - f s}$ driving pulses with controlled electric field and peak intensity beyond the gas saturation intensity. The availability of attosecond sources with high peak intensities has potential in opening new avenues for attosecond-pump/attosecond-probe studies of electronic processes in atomic and molecular physics, with interesting prospects in the field of coherent control of electronic motion in complex systems in the attosecond temporal regime.
\end{abstract}

A lmost ten years after the first experimental demonstration of the generation of isolated attosecond pulses ${ }^{1}$, attosecond technology has become an important branch of ultrafast science, with important technological and scientific breakthroughs ${ }^{2,3}$. Isolated attosecond pulses have been generated using three different approaches based on high-order harmonic generation (HHG) in gases, and produced by few-optical-cycle driving pulses. The first approach is based on the use of sub-5-fs pulses with a stabilized carrier-envelope phase (CEP) and spectral selection of the cutoff portion of the harmonic spectrum ${ }^{4}$. Pulses as short as 80 as at a photon energy of $\sim 80 \mathrm{eV}$ were generated with this method in 2008 (ref. 5). The second approach makes use of phase-stabilized few-cycle driving pulses in combination with the polarization gating technique ${ }^{6-9}$, resulting in the generation of completely characterized pulses as short as 130 as at a photon energy of $\sim 36 \mathrm{eV}$ (ref. 10). The third method relies on a temporal gating technique based on the ionization-induced phase mismatching between the fundamental and harmonic fields. Using this technique, 430-as pulses at $\sim 90 \mathrm{eV}$ were generated (ref. 11). Isolated attosecond pulses have also been generated using multi-optical-cycle pulses (with durations up to $28 \mathrm{fs}$ ) by taking advantage of the generalized double optical gating (GDOG) method, which is a generalized polarization gating technique based on the use of a two-colour driving field ${ }^{12,13}$. In parallel with the development of attosecond science based on isolated attosecond pulses, remarkable advances have also been achieved in the generation and application of trains of attosecond pulses, with important progress in attosecond metrology ${ }^{14-16}$ and the demonstration of experimental techniques based on the use of trains of attosecond pulses ${ }^{17}$.

To date, measurements with attosecond temporal resolution have been performed by using an isolated attosecond pulse as pump and an infrared (IR) femtosecond pulse as probe, or vice versa. A real breakthrough in attosecond science would be the ability to probe with isolated attosecond pulses the electron dynamics initiated by an attosecond pump pulse. The crucial limitation that has so far prevented the demonstration of attosecond-pump/attosecond-probe spectroscopy is the extremely low pulse energy of the available attosecond sources. By using the technique of spectral selection of the cutoff harmonics in combination with the use of almost single-cycle excitation pulses, $\sim 1 \times 10^{8}$ photons/s (photon energy, $\sim 80 \mathrm{eV}$ ) on target have been reported (by using neon as the generating medium), which corresponds to a pulse energy of $\sim 0.5 \mathrm{pJ}$ (ref. 5). In this case the pulse energy at the output of the gas cell used for HHG $(\sim 0.5 \mathrm{~nJ})$ is reduced by three orders of magnitude by the multilayer mirror used for spectral selection and by the zirconium foil used to eliminate the fundamental and lower harmonic orders. By using the polarization gating $(\mathrm{PG})$ technique, $\sim 70-\mathrm{pJ}$ (on target) attosecond pulses at $\sim 35 \mathrm{eV}$ photon energy have been generated in $\operatorname{argon}^{8}$.

Here, we demonstrate a technique for the generation of isolated attosecond pulses with energy up to $2.1 \mathrm{~nJ}$ on target, thus showing a pulse-energy enhancement from one to three orders of magnitude compared with the generating methods demonstrated so far. The key elements of the technique are (i) the use of few-optical-cycle driving pulses with a controlled electric field, linear polarization and peak intensity above the saturation intensity of the gas used for HHG, defined as the peak intensity leading to an ionization probability of 1 , and (ii) a careful design of the interaction geometry in terms of gas pressure, position and thickness of the gas cell. We will demonstrate that such a method is based on the combined action of complete depletion of the neutral atom population on the leading edge of the few-cycle excitation pulse ${ }^{18}$ and efficient spatial filtering in the extreme ultraviolet (XUV) beamline.

\section{Results}

Spectral and temporal characterization of the attosecond pulses. We used 5-fs driving pulses with a controlled electric field to generate XUV radiation by HHG in a $2.5-\mathrm{mm}$-long cell filled with xenon at static pressure $(\sim 2.5-3$ torr $)$. Figure la shows the XUV spectra obtained at a laser peak intensity of $I \approx(2.3 \pm 0.3) \times$ $10^{15} \mathrm{~W} \mathrm{~cm}^{-2}$ in the beam waist position: well-resolved harmonic peaks are clearly visible, ranging from the 13th harmonic up to the 23rd harmonic (note that the saturation intensity of xenon is $\sim 3.7 \times 10^{14} \mathrm{~W} \mathrm{~cm}^{-2}$ for 5 -fs excitation pulses). Upon changing the CEP of the driving pulses by $\Delta \psi=\pi / 2$, a clear transition from the harmonic structure shown in Fig. 1a to a continuous spectrum is observed (Fig. 1b). This is a strong evidence that, in the temporal domain, by changing the CEP of the driving pulses it is possible to confine the XUV generation process to a single event. Continuous spectra can be generated within a broad range 

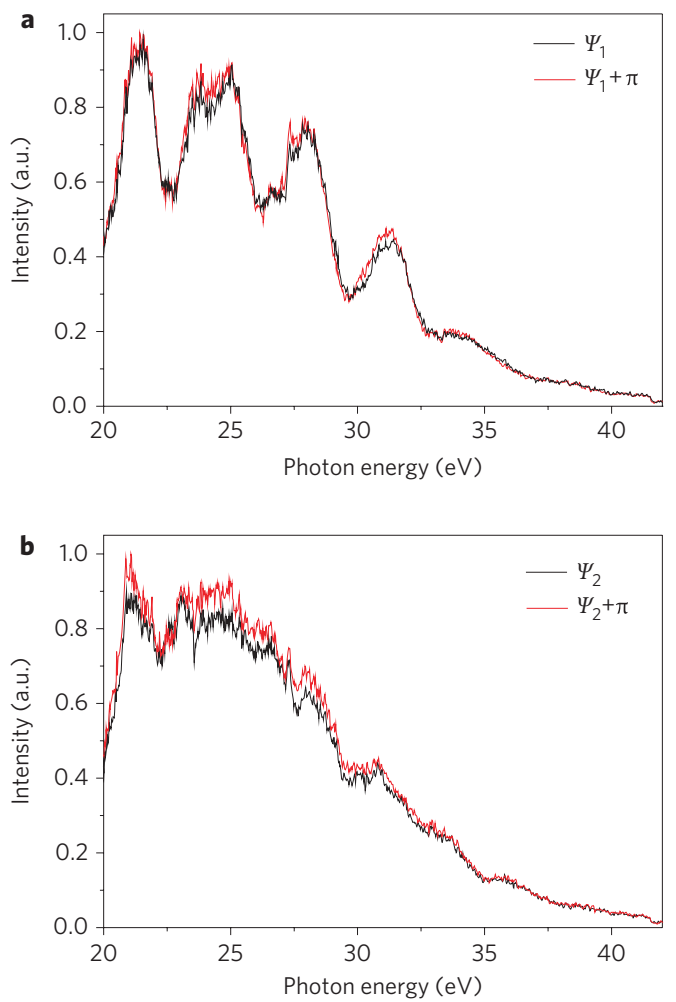

Figure 1 | CEP-dependence of measured XUV spectra. XUV spectra generated in a 2.5 -mm-thick xenon cell by 5 -fs pulses at a peak intensity $I \approx(2.3 \pm 0.3) \times 10^{15} \mathrm{~W} \mathrm{~cm}^{-2}$ for different CEP values: $\psi_{1}, \psi_{1}+\pi(\mathbf{a})$, $\psi_{2}, \psi_{2}+\pi(\mathbf{b})$, where $\psi_{1}=\psi_{2}+\pi / 2$. The spectra were measured after the aluminium filter used to block the fundamental radiation and the low-order harmonics.

of CEP values, much larger than the residual CEP fluctuations after stabilization. The spectral shapes periodically change for a CEP variation $\Delta \psi=\pi$. Indeed, both Fig. $1 \mathrm{a}$ and $1 \mathrm{~b}$ show two XUV spectra acquired for a CEP variation $\Delta \psi=\pi$. The energy of the XUV pulses in the case of the continuous spectra was $2.1 \mathrm{~nJ}$, after a 100-nm-thick aluminium filter used to block the fundamental radiation and the low-order harmonics. Therefore, this is the XUV energy on target, which can really be used in an experiment. Such an energy corresponds to $\sim 5.3 \times 10^{8}$ photons/pulse (or $\sim 5.3 \times 10^{11}$ photons/s). Continuous XUV spectra were obtained in a relatively broad interval, $\Delta z \approx 2 \mathrm{~mm}$, of gas cell positions from the focus of the laser beam. The optimal gas pressure was below the maximum value leading to absorption of the XUV radiation. We have also used krypton, argon and neon as generating media. In such cases a clear transition from modulated to continuous XUV spectra was observed upon changing the CEP of the driving pulses. In particular, in neon it was possible to generate continuous spectra extending from $65 \mathrm{eV}$ to $160 \mathrm{eV}$, with a maximum energy of $230 \mathrm{pJ}$ on target.

To measure the temporal characteristics of the attosecond pulses, we used the method of frequency resolved optical gating for complete reconstruction of attosecond bursts (FROG CRAB) ${ }^{19}$. The XUV pulses transmitted by the aluminium plate were focused onto an argon gas jet, where they ionized the gas atoms in the presence of an IR pulse (hereafter called the streaking pulse), which acted as an ultrafast phase modulator on the generated electron wave packet. By measuring the temporal evolution of the photoelectron spectra as a function of the delay between the XUV pulse and the streaking pulse it is possible to retrieve the temporal characteristics of the attosecond pulse. Figure 2 a shows a portion of the
FROG CRAB trace measured for XUV pulses generated in xenon by 5 -fs pulses with a CEP value leading to the production of a continuous spectrum. The retrieved FROG CRAB trace, shown in Fig. $2 b$, reproduces the experimental trace; the corresponding error, evaluated as the root-mean-square error per element of the trace ${ }^{20}$, is $\sim 1 \times 10^{-3}$. In the reconstructed temporal intensity profile of the XUV pulses, reported in Fig. 2c, the pulse duration was $155 \pm 5$ as (the transform limit was $\sim 130$ as). Such pulses correspond to only 1.1 optical cycles of the carrier frequency.

Physical mechanism: nonadiabatic saddle-point simulations. The physical mechanism underlying the confinement of XUV generation to a single event by using high-peak-intensity driving pulses with fewoptical-cycle duration is related to the ionization dynamics in the generating medium. The role of ionization in the generation of isolated attosecond pulses has already been investigated for 8 -fs (ref. 11) and 15-fs (ref. 21) driving pulses with different experimental setups. In both cases, the confinement of the XUV generation process to a single event was restricted to the cutoff region of the XUV spectrum, and it was explained by the very rapid loss of phase matching occurring on the leading edge of the excitation pulses. The use of intense CEP-stabilized few-cycle pulses for the generation of broadband, continuous XUV radiation has been discussed in earlier theoretical works ${ }^{22,23}$. In both cases, the generation of isolated attosecond pulses required a suitable spectral selection of the high-frequency components of the XUV spectrum. In contrast, in our experimental configuration, both the plateau and cutoff regions of the XUV spectrum are continuous. The fundamental radiation and the low-order harmonics have been blocked by a thin metallic filter.
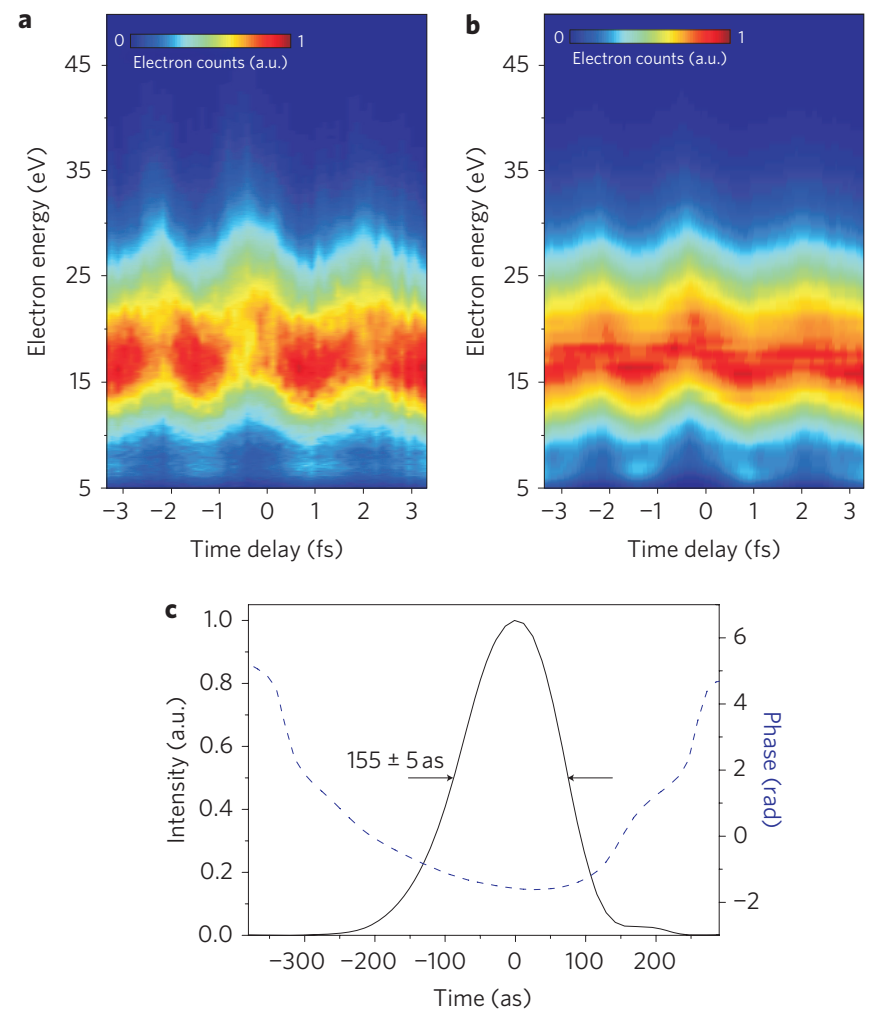

Figure 2 | FROG CRAB measurement of isolated attosecond pulses. a, Portion of an experimental FROG CRAB trace as a function of the temporal delay between the attosecond and streaking pulses. b. Reconstruction of the FROG CRAB trace after $8 \times 10^{5}$ iterations of the retrieval algorithm. $\mathbf{c}$, Reconstruction of the temporal intensity profile and phase of the attosecond pulses. 

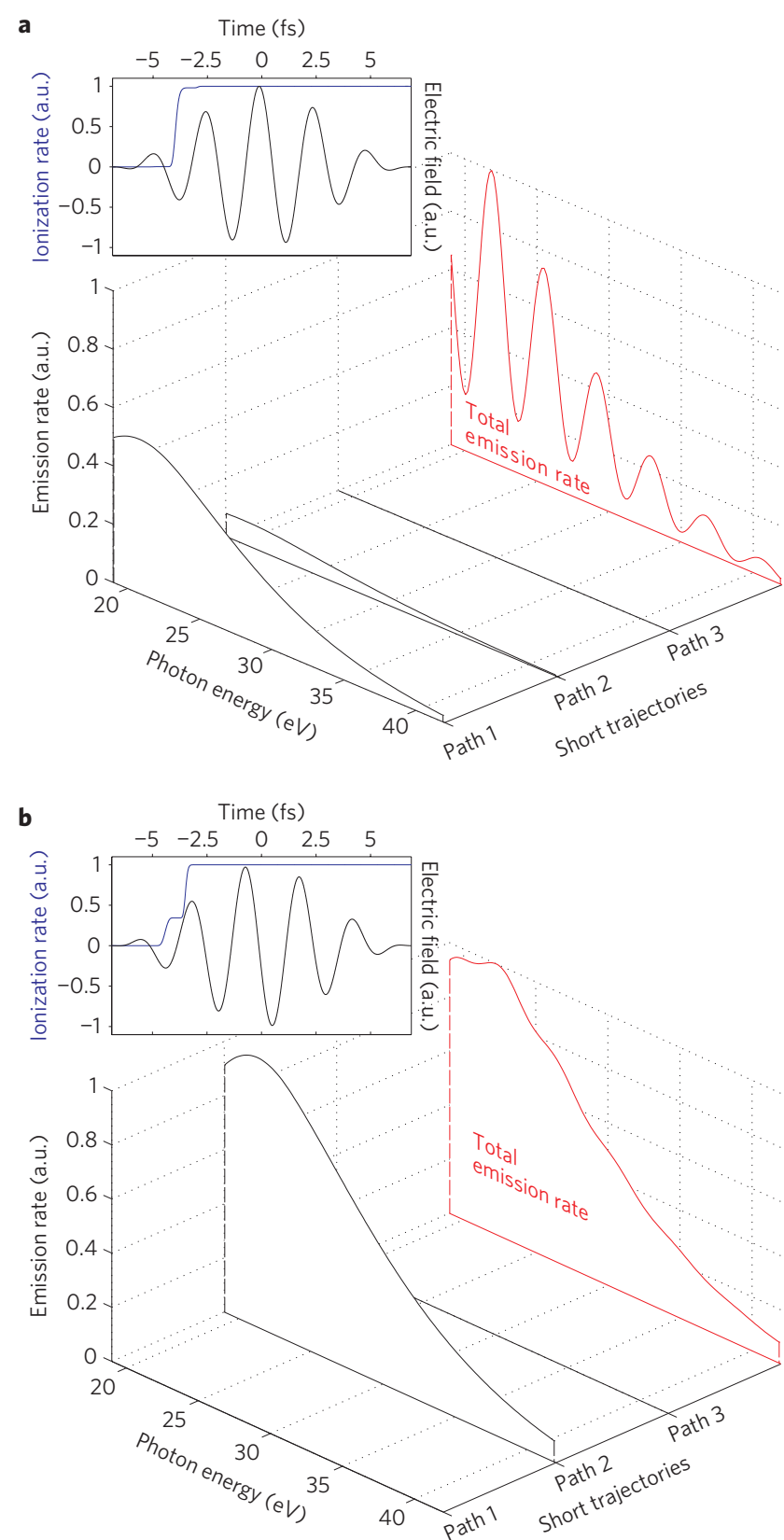

Figure 3 | Quantum path contributions to the XUV emission rate by saddle-point simulations. $\mathbf{a}, \mathbf{b}, \mathrm{XUV}$ emission rates associated with the first three short quantum paths (blue curves) and total XUV emission rate produced by coherent superposition of all short electron quantum paths (red curve) for two CEP values: $\psi=0.1 \pi(\mathbf{a})$ and $0.6 \pi(\mathbf{b})$. The contribution of the aluminium filter has been taken into account. Insets electric field of the driving pulses and corresponding ionization rates. Parameters used in the nonadiabatic saddle-point simulations: laser central wavelength, $750 \mathrm{~nm}$; pulse duration, $5 \mathrm{fs}$; pulse peak intensity, $2.5 \times 10^{15} \mathrm{~W} \mathrm{~cm}^{-2}$; xenon gas.

To obtain a first direct physical interpretation of the physical processes underlying the experimental results, we used the nonadiabatic saddle-point method ${ }^{24,25}$, which analyses the XUV generation process in terms of the complex trajectories (quantum paths) followed by the electrons from the ionization instant to the recombination with the parent ion. It is well known that on-axis emission is predominantly related to the so-called short quantum paths, characterized by an electron return time of the order of one-half an optical period of the fundamental radiation ${ }^{26}$. We calculated the contribution to the total XUV emission rate of each individual short quantum path, taking into account the ground-state depletion by using Ammosov, Delone and Krainov (ADK) theory ${ }^{27}$, as described in ref. 28. Figure 3 a shows the XUV emission rates associated with the first three quantum paths for a CEP value of $\psi=0.1 \pi$. As a result of the particular temporal evolution of the ionization rate (inset, Fig. 3a), two quantum paths contribute to the total emission rate (red curve in Fig. 3a), which was found to be spectrally modulated. In such a case, two attosecond pulses are generated. On changing the CEP value to $\pi / 2$, the fast depletion of the neutral atom population (inset, Fig. 3b) leads to the confinement of the generation process to a single quantum path (thus giving rise to an isolated attosecond pulse), as is clearly shown in Fig. 3b. We then verified that the XUV generation process was not affected by multiple ionization of the generating medium (see Supplementary Information). Of course, the saddle-point method does not consider the propagation effects and the radial intensity variation of the driving field. Because the generation mechanism being used is crucially determined by the evolution of the neutral atom population density, which is directly related to the driving intensity, it is particularly important to use a nonadiabatic three-dimensional numerical propagation model $^{29}$.

Nonadiabatic three-dimensional propagation model. The singleatom response was used in a three-dimensional propagation model, which takes into account both temporal plasma-induced phase modulation and spatial plasma-lensing effects on the
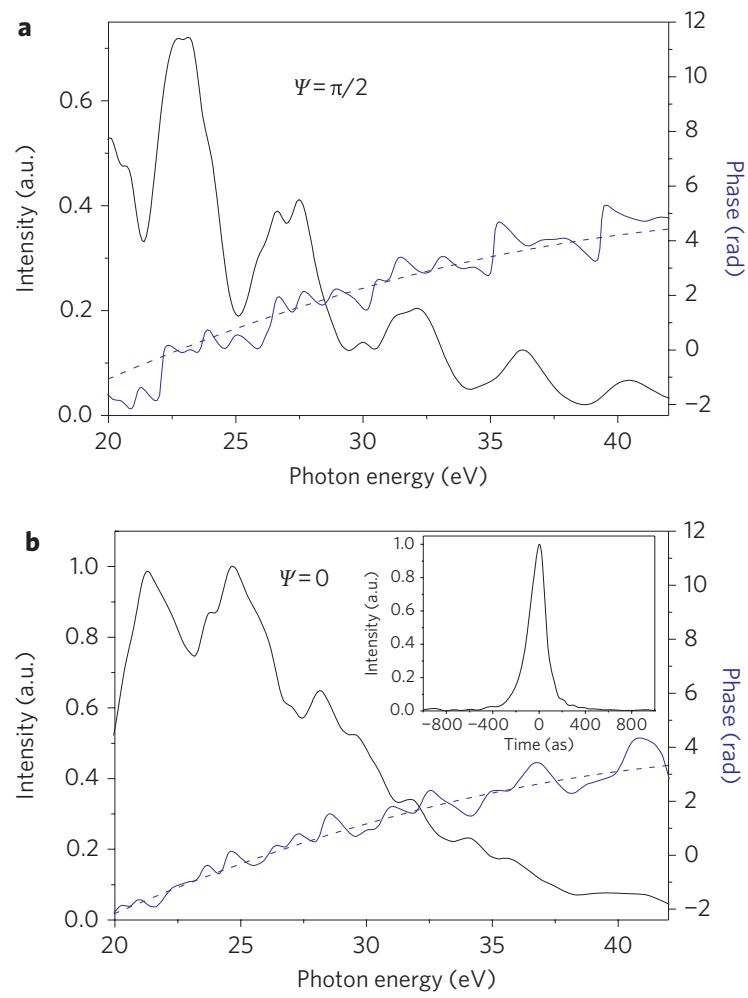

Figure 4 | Calculated spectral and temporal characteristics of XUV radiation. $\mathbf{a}, \mathbf{b}$ XUV spectra in the focal plane of a toroidal mirror (target position) calculated using the three-dimensional numerical model (discussed in the text), for the same driving pulse parameters and interaction geometry used in the experiment and two different CEP values: $\psi=\pi / 2$ (a) and (b). Solid blue curves are the calculated spectral phases; dashed blue lines are parabolic spectral phases with a positive group-delay dispersion $\phi^{\prime \prime}=3 \times 10^{3}$ as $^{2}$. Inset to panel $\mathbf{b}$ : calculated temporal intensity profile of the isolated attosecond pulses for $\psi=0$. A pulse duration of 160 as was calculated, taking into account the contribution of a 120 -nm-thick aluminium filter. 

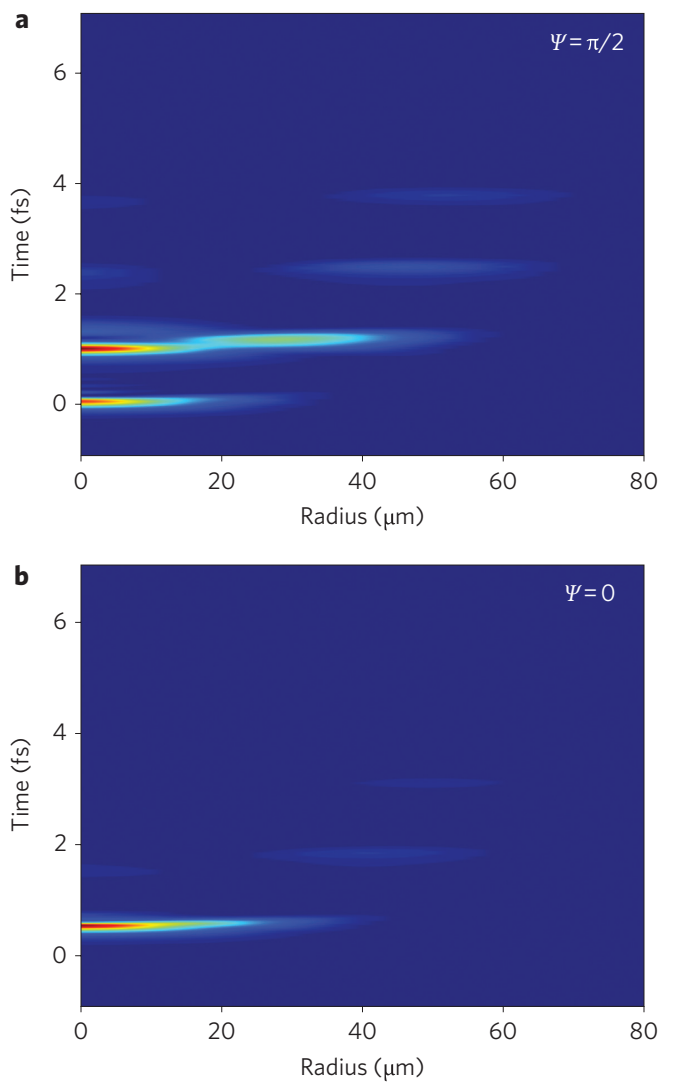

Figure $\mathbf{5}$ | Spatiotemporal characteristics of attosecond pulses.

$\mathbf{a}, \mathbf{b}$, Temporal evolution of XUV pulses as a function of the radial coordinate, calculated in the target position using the three-dimensional numerical model (discussed in the text), with the same parameters used in Fig. 4. Two CEP values were considered: $\psi=\pi / 2$ (a) and 0 (b).

fundamental beam. In agreement with the experimental results, the spatial reshaping of the driving pulse was found to be weak, also considering multiple ionization of the generating medium, as a result of the very low pressure used in the experiment. Figure 4 shows two XUV spectra calculated in the focal plane of the toroidal mirror used to focus the XUV radiation (hereafter, such a position will be called the target position), for the same driving pulse parameters and interaction geometry used in the experiment. In agreement with the experimental results, the XUV spectra display an evolution from a modulated behaviour $(\psi=\pi / 2$, Fig. $4 \mathrm{a})$ to a continuous one $(\psi=0$, Fig. $4 \mathrm{~b})$ on changing the CEP value. We checked that such spectral features were not dramatically influenced by the gas cell position in a relatively large range $\Delta z \approx 2 \mathrm{~mm}$ from the focal plane of the driving radiation. The calculated spectral phases $\phi(\omega)$ (also shown in Fig. 4) are characterized by an almost parabolic shape with a positive chirp $\phi^{\prime \prime}=3 \times 10^{3}$ as $^{2}$ (the dashed lines are parabolic fitting curves).

We then investigated the spatial characteristics of the XUV radiation. The spatial distribution of the driving laser intensity could give rise to XUV pulses with inhomogeneous temporal characteristics across the beam transverse profile. Figure 5 displays the temporal evolution of the XUV pulses as a function of the radial coordinate, calculated in the target position for two CEP values; the colour scale represents the XUV peak intensity. We calculated the propagation of the XUV beam from the gas cell to the target position by taking into account the effects of the toroidal mirror and a 200- $\mu \mathrm{m}$-diameter pinhole used for spatial filtering of the XUV radiation. For the case of $\psi=\pi / 2$ (Fig. 5a), the XUV emission is characterized by two attosecond pulses, for which the relative peak intensity changes with the radial coordinate; this is in agreement with the observation of a modulated XUV spectrum for such a CEP value. For the case $\psi=0$ (see Fig. 5b), isolated attosecond pulses are generated, with a constant pulse duration across the transverse profile of the beam $(\sim 200$ as without chirp compensation), thus demonstrating the excellent spatial characteristics of the generated attosecond pulses. The inset in Fig. $4 \mathrm{~b}$ shows the calculated temporal intensity profile of the isolated attosecond pulses. A pulse duration of 160 as was calculated when considering the contribution of a 120-nm-thick aluminium filter (we assumed a constant group delay dispersion $\phi^{\prime \prime}=-3 \times 10^{3} \mathrm{as}^{2}$, according to the experimental results reported in ref. 30 . To obtain experimental information about the spatiotemporal characteristics of the XUV pulses we placed a horizontal slit with a $200-\mu \mathrm{m}$ aperture in front of the XUV spectrometer ( $\sim 40 \mathrm{~cm}$ after the target position) and moved it across the XUV beam in the vertical direction. When choosing the CEP value for a continuous spectrum, we observed that the spectral characteristics of the XUV pulses were the same for each position of the slit. This is strong experimental evidence of the excellent spatial quality of the generated XUV radiation.

\section{Discussion}

The energy of the attosecond pulses reported in this work was limited by the energy of the few-cycle driving pulses. With appropriate choice of the interaction geometry (for example, by loose focusing of the excitation pulses), the technique might be scaled to higher energies. Moreover, to increase the peak intensity of the isolated attosecond pulses it is possible to optimize the focusing geometry of the XUV radiation. Indeed, by using a platinum-coated ellipsoidal mirror, Others were able to focus intense coherent light in the spectral region $25-40 \mathrm{~nm}$ into a $2.4-\mu \mathrm{m}$ spot size $\mathrm{e}^{31}$. Assuming such a focusing geometry for the attosecond pulses reported in this work, a XUV peak intensity of $7.2 \times 10^{13} \mathrm{~W} \mathrm{~cm}^{-2}$ could be obtained on target, which is larger than the minimum peak intensity required to induce two-photon transitions in the XUV region $\left(\sim 10^{12} \mathrm{~W} \mathrm{~cm}^{-2}\right)^{16}$. We can foresee various important applications of such intense attosecond pulses. Attosecond metrology based on nonlinear XUV optics ${ }^{18}$ would greatly benefit from an increase in the energy of isolated attosecond pulses. A significant breakthrough would be the realization of attosecond-pump/attosecond-probe measurements, to obtain direct information on the subfemtosecond dynamics initiated by attosecond pulses focused on atomic, molecular or solid samples. Of particular interest are applications for the observation and control, on an attosecond temporal scale, of electron wave-packet dynamics in biomolecules ${ }^{32}$. The use of the attosecond-pump/attosecond-probe scheme could allow the development of experimental techniques designed to achieve coherent control of electronic motion in complex systems on a purely electronic scale. The investigation and control of attosecond electron dynamics in biomolecules has the potential to open new avenues in the field of biophysics, because the electron migration process in large molecules forms the basis of fundamental reactions in biology. A crucial step in this regard is the generation of an isolated attosecond pulse with energy at least in the nanojoule range, as has been demonstrated in this work.

Received 8 March 2010; accepted 29 September 2010; published online 14 November 2010

\section{References}

1. Hentschel, M. et al. Attosecond metrology. Nature 414, 509-513 (2001).

2. Krausz, F. \& Ivanov, M. Attosecond physics. Rev. Mod. Phys. 81, 163-234 (2009).

3. Nisoli, M. \& Sansone, S. New frontiers in attosecond science. Prog. Quantum Electron. 33, 17-59 (2009). 
4. Christov, I. P., Murnane, M. M. \& Kapteyn, H. High-harmonic generation of attosecond pulses in the 'single-cycle' regime. Phys. Rev. Lett. 78, 1251-1254 (1997).

5. Goulielmakis, E. et al. Single-cycle nonlinear optics. Science 320, 1614-1617 (2008).

6. Corkum, P. B., Burnett, N. H. \& Ivanov, M. Y. Subfemtosecond pulses. Opt. Lett. 19, 1870-1872 (1994).

7. Tcherbakoff, O., Mével, E., Descamps, D., Plumridge, J. \& Constant, E. Timegated high-order harmonic generation. Phys. Rev. A 68, 043804 (2003).

8. Sola, I. J. et al. Controlling attosecond electron dynamics by phase-stabilized polarization gating. Nature Phys. 2, 319-322 (2006).

9. Mashiko, H. et al. Double optical gating of high-order harmonic generation with carrier-envelope phase stabilized lasers. Phys. Rev. Lett. 100, 103906 (2008).

10. Sansone, G. et al. Isolated single-cycle attosecond pulses. Science 314, 443-446 (2006).

11. Abel, M. J. et al. Isolated attosecond pulses from ionization gating of highharmonic emission. Chem. Phys. 366, 9-14 (2009).

12. Feng, X. et al. Generation of isolated attosecond pulses with 20 to 28 femtosecond lasers. Phys. Rev. Lett. 103, 183901 (2009).

13. Gilbertson, S., Khan, S. D., Wu, Y., Chini, M. \& Chang, Z. Isolated attosecond pulse generation without the need to stabilize the carrier-envelope phase of driving lasers. Phys. Rev. Lett. 105, 093902 (2010).

14. Paul, P. M. et al. Observation of a train of attosecond pulses from high harmonic generation. Science 292, 1689-1692 (2001)

15. Tzallas, P., Charalambidis, D., Papadogiannis, N. A., Witte, K. \& Tsakiris, G. D. Direct observation of attosecond light bunching. Nature 426, 267-271 (2003).

16. Midorikawa, K., Nabekawa, Y. \& Suda, A. XUV multiphoton processes with intense high-order harmonics. Prog. Quantum Electron. 32, 43-88 (2008)

17. Remetter, T. et al. Attosecond electron wave packet interferometry. Nature Phys. 2, 323-326 (2006).

18. Sekikawa, T., Kosuge, A., Kanai, T. \& Watanabe, S. Nonlinear optics in the extreme ultraviolet. Nature 432, 605-608 (2004).

19. Mairesse, Y. \& Quéré, F. Frequency-resolved optical gating for complete reconstruction of attosecond bursts. Phys. Rev. A 71, 011401(R) (2005).

20. Delong, K. W., Fittinghoff, D. N. \& Trebino, R. Practical issues in ultrashortlaser-pulse measurement using frequency-resolved optical gating. IEEE J. Quantum Electron. 32, 1253-1264 (1996).

21. Thomann, I. et al. Characterizing isolated attosecond pulses from hollow-core waveguides using multi-cycle driving pulses. Opt. Express 17, 4611-4633 (2009).

22. Cao, W. et al. Single-attosecond pulse generation with an intense multicycle driving pulses. Phys. Rev. A 74, 063821 (2006)

23. Kim, K. T., Kim, C. M., Baik, M. G., Umesh, G. \& Nam, C. H. Single sub-50attosecond pulse generation from chirp-compensated harmonic radiation using material dispersion. Phys. Rev. A 69, 051805(R) (2004).
24. Lewenstein, M., Balcou, Ph., Ivanov, M. Y., L'Huillier, A. \& Corkum, P. B. Theory of high-harmonic generation by low-frequency laser pulses. Phys. Rev. A 49, 2117-2132 (1994)

25. Sansone, G., Vozzi, C., Stagira, S. \& Nisoli, M. Nonadiabatic quantum path analysis of high-order harmonic generation: role of the carrier-envelope phase on short and long paths. Phys. Rev. A. 70, 013411 (2004).

26. Bellini, M. et al. Temporal coherence of ultrashort high-order harmonic pulses. Phys. Rev. Lett. 81, 297-300 (1998).

27. Ammosov, M. V., Delone, N. B. \& Krainov, V. P. Zh. Eksp. Teor. Fiz. 91, 2008 (1986). [Sov. Phys. JETP 64, 1191 (1986)].

28. Sansone, G. et al. Control of long electron quantum paths in high-order harmonic generation by phase-stabilized light pulses. Phys. Rev. A 73, 053408 (2006)

29. Priori, E. et al. Nonadiabatic three-dimensional model of high-order harmonic generation in the few-optical-cycle regime. Phys. Rev. A 61, 63801 (2000).

30. López-Martens, R. et al. Amplitude and phase control of attosecond light pulses. Phys. Rev. Lett. 94, 033001 (2005).

31. Mashiko, H., Suda, A. \& Midorikawa, K. Focusing multiple high-order harmonics in the extreme-ultraviolet and soft-X-ray regions by a platinumcoated ellipsoidal mirror. Appl. Opt. 45, 573-577 (2006).

32. Remacle, F. \& Levine, R. D. An electronic time scale in chemistry. Proc. Nat Acad. Sci. USA 103, 6793-6798 (2006).

\section{Acknowledgements}

The research leading to the results presented in this paper was supported by funding from the European Research Council under the European Community's Seventh Framework Programme (FP7/2007-2013)/ERC grant agreement no. 227355 - ELYCHE. The authors acknowledge financial support from the Italian Ministry of Research (FIRB-IDEAS RBID08CRXK), support from European Union under contract no. 228334 JRA-ALADIN (Laserlab Europe II) and from MC-RTN ATTOFEL (FP7-238362)

\section{Author contributions}

F.F., F.C. and M.L. contributed equally to this work and performed the experiments. F.C. performed the three-dimensional simulations, M.L. performed the saddle-point simulations and F.F. and M.L. performed the FROG CRAB simulations. C.V. and S.S. contributed to the development of the experimental setup. G.S. was responsible for the construction of the attosecond cross-correlation setup. M.N. supervised the project and wrote the manuscript.

\section{Additional information}

The authors declare no competing financial interests. Supplementary information accompanies this paper at www.nature.com/naturephotonics. Reprints and permission information is available online at http://npg.nature.com/reprintsandpermissions/. Correspondence and requests for materials should be addressed to M.N. 